\title{
Pressure effects on the magnetic transition temperature in ordered double perovskites
}

\author{
D. Di Castro, ${ }^{1,2}$ P. Dore, ${ }^{2}$ R. Khasanov, ${ }^{3,4}$ H. Keller, ${ }^{3}$ P. Mahadevan, ${ }^{5}$ Sugata Ray, ${ }^{6,7}$ D. D. Sarma,, 8 and P. Postorino ${ }^{2}$ \\ ${ }^{1}$ CNR-INFM Coherentia and Department of Mechanical Engineering, University of Rome "Tor Vergata," Via del Politecnico 1, \\ I-00133 Rome, Italy \\ ${ }^{2}$ CNR-INFM Coherentia and Dipartimento di Fisica, Università di Roma "La Sapienza," P.le A. Moro 2, I-00185 Rome, Italy \\ ${ }^{3}$ Physik-Institut der Universität Zürich, Winterthurerstrasse 190, CH-8057 Zürich, Switzerland \\ ${ }^{4}$ Laboratory for Muon Spin Spectroscopy, Paul Scherrer Institut, CH-5232 Villigen PSI, Switzerland \\ ${ }^{5}$ S.N. Bose National Center for Basic Sciences, JD Block, Sector III, Kolkata 700098, India \\ ${ }^{6}$ Solid State and Structural Chemistry Unit, Indian Institute of Science, Bangalore 560012, India \\ ${ }^{7}$ Department of Materials Science, Indian Association for the Cultivation of Science, Kolkata 700032, India \\ ${ }^{8}$ Centre for Advanced Materials, Indian Association for the Cultivation of Science, Kolkata 700032, India
}

(Received 10 June 2008; revised manuscript received 17 October 2008; published 17 November 2008)

\begin{abstract}
A study of the pressure effect on the Curie temperature in polycrystalline ordered double perovskites was carried out by measuring the temperature dependence of the magnetization in isoelectronic $\mathrm{Sr}_{2} \mathrm{FeMoO}_{6}$, $\mathrm{Ba}_{2} \mathrm{FeMoO}_{6}$, and $\mathrm{Ca}_{2} \mathrm{FeMoO}_{6}$, and in W-substituted $\mathrm{Sr}_{2} \mathrm{FeMo}_{1-x} \mathrm{~W}_{x} \mathrm{O}_{6}$ with $x=0.70$. Pressure up to approximately $10 \mathrm{kbar}$ induces an increase in the Curie temperature in all the samples with different rate: $\mathrm{d} T_{c} / \mathrm{dp}$ results to be 1.7(2) $\mathrm{K} / \mathrm{kbar}$ for $\mathrm{Ba}_{2} \mathrm{FeMoO}_{6}, 0.60(8) \mathrm{K} / \mathrm{kbar}$ for $\mathrm{Sr}_{2} \mathrm{FeMoO}_{6}, 0.25(8) \mathrm{K} / \mathrm{kbar}$ for $\mathrm{Ca}_{2} \mathrm{FeMoO}_{6}$, and $0.7(1) \mathrm{K} / \mathrm{kbar}$ for the $\mathrm{W}$-substituted sample. The results are interpreted in terms of the different lattice structure and cell volume of the investigated systems. A manganitelike model was only partially successful in explaining the observed pressure behavior, thus, pointing out the different nature of the magnetic interaction in doped manganites and in ordered double perovskites.
\end{abstract}

DOI: 10.1103/PhysRevB.78.184416

PACS number(s): 75.47.Gk, 62.50.-p, 51.60.+a

\section{INTRODUCTION}

The ordered double-perovskite materials $A_{2} B B^{\prime} \mathrm{O}_{6}$, with $A$ as an alkaline earth, such as $\mathrm{Sr}, \mathrm{Ba}$, or $\mathrm{Ca}$, and $B$ and $B^{\prime}$ as two different transition metals, are interesting for several aspects. In $\mathrm{Sr}_{2} \mathrm{FeMoO}_{6}$ the high Curie temperature $\left(T_{C}\right.$ $\simeq 400 \mathrm{~K}$ ) was known since the $1960 \mathrm{~s},{ }^{1}$ whereas colossal magnetoresistance (CMR) at temperatures higher and magnetic fields lower than in other systems (e.g., doped manganites ${ }^{2}$ ) was recently discovered by Kobayashi et al. ${ }^{3}$ These characteristics make $\mathrm{Sr}_{2} \mathrm{FeMoO}_{6}$ and, more general, double-perovskite systems promising materials for roomtemperature technological applications. ${ }^{4}$

Fundamental progresses have been made to understand the physics of the double perovskites, ${ }^{5}$ and theoretical models were developed. ${ }^{5,6}$ In order to better understand the interplay between structural, electronic, and magnetic degrees of freedom, systematic studies of $A A^{\prime} B B^{\prime} \mathrm{O}_{6}$ systems, with different alkaline earth $A$ and $A^{\prime}$ atoms, ${ }^{6-8}$ and transition metal $B$ and $B^{\prime}$ atoms ${ }^{9-12}$ were performed by several authors. Among these compounds, the $\mathrm{Sr}_{2-x} A_{x}^{\prime} \mathrm{FeMoO}_{6}$ families, with $A^{\prime}=\mathrm{Ba}$ and $\mathrm{Ca}$, were widely studied. ${ }^{1,6-8,13-22}$ Structural data obtained on these isoelectronic systems show that $\mathrm{Ba}_{2} \mathrm{FeMoO}_{6}$ has an ideal cubic structure, whereas, on increasing the chemical pressure by decreasing the $A$-ion size from $\mathrm{Ba}$ to $\mathrm{Sr}$ and $\mathrm{Ca}$, the lattice structure changes to tetragonal (pseudocubic) in $\mathrm{Sr}_{2} \mathrm{FeMoO}_{6}$, with the $\mathrm{Fe}-\mathrm{O}-\mathrm{Mo}$ bond angle of about $174^{\circ}$, and to monoclinic in $\mathrm{Ca}_{2} \mathrm{FeMoO}_{6}$, with bond angle $\simeq 152^{\circ} .8$

A systematic study of the effects induced by chemical pressure on $T_{C}$ and $\mathrm{CMR}$ in $\mathrm{Sr}_{2} \mathrm{FeMoO}_{6}$, by the gradual substitution of $\mathrm{Sr}$ with $\mathrm{Ca}$ and $\mathrm{Ba}$, was performed by Kim et al. ${ }^{7}$ A nonmonotonic evolution of CMR and $T_{C}$ with chemical pressure was observed. The highest value of low-field CMR was found in the $\mathrm{Ba}$ substituted $\mathrm{Sr}_{2-x} \mathrm{Ba}_{x} \mathrm{FeMoO}_{6}$ (at $x=1.6$ ), whereas the highest $T_{C}$ was observed at small Ca concentration in $\mathrm{Sr}_{2-x} \mathrm{Ca}_{x} \mathrm{FeMoO}_{6}$ (at $x=0.1$ ). The evolution of $T_{C}$ with chemical pressure was interpreted in terms of bond lengths $\left(B-\mathrm{O}\right.$ and $\left.B^{\prime}-\mathrm{O}\right)$ and bond angle $\left(B-\mathrm{O}-B^{\prime}\right)$ modifications, and the consequent change in electronic bandwidth (BW) and electron hopping. ${ }^{7,8}$

Not only the substitution of alkaline metal $(A)$ changes the physical properties of double perovskites. The substitution of the transition-metal ions $\left(B, B^{\prime}\right)$ leads to the observation of even more dramatic changes in the electronic and magnetic properties. For example, the substitution of Mo with $\mathrm{W}$ ions in $\mathrm{Sr}_{2} \mathrm{FeMo}_{1-x} \mathrm{~W}_{x} \mathrm{O}_{6}$, which introduces a charge doping, does not modify the crystal structure (tetragonal at all $\mathrm{W}$ concentrations), but induces a negative chemical pressure by expanding the cell volume, and strongly changes the electronic and magnetic properties. ${ }^{9-12,23}$ It was shown ${ }^{9,10,12}$ that a ferromagnetic moment survives up to $x=0.9$, with $T_{C}$ strongly decreasing with increasing $x$, and that an insulating spin-glass behavior is found for $x>0.75$. An insulating antiferromagnetic state is finally established in the $x=1$ pristine $\mathrm{Sr}_{2} \mathrm{FeWO}_{6}$ below about $35 \mathrm{~K}^{9}{ }^{9-11}$

Similarly to the chemical pressure, the application of an external pressure can induce changes of bond lengths and angles. Compared to the chemical pressure, the external pressure is a clean tool to avoid substitutional induced disorder and spurious effects due to the use of many samples with different chemical composition. However, in magnetic measurements the maximum applied pressure is generally limited to $10-20 \mathrm{kbar}$, and the corresponding variation in $\mathrm{BW}$ is typically smaller than that obtained by proper chemical substitution. More information can be, thus, obtained from a combined use of both chemical and external pressure. 
In the present work we performed a systematic investigation of the evolution of $T_{C}$ with external pressure in several double perovskites, which, at ambient pressure, exhibit different chemical pressure. Magnetization measurements under pressure were performed on $\mathrm{Sr}_{2} \mathrm{FeMoO}_{6}, \mathrm{Ba}_{2} \mathrm{FeMoO}_{6}$, $\mathrm{Ca}_{2} \mathrm{FeMoO}_{6}$, and $\mathrm{Sr}_{2} \mathrm{FeMo}_{1-x} \mathrm{~W}_{x} \mathrm{O}_{6}$ with $x=0.7$ samples. In all cases an increase in $T_{C}$ was found on increasing pressure, but with different rates. Similar measurements were also performed on $\mathrm{Sr}_{2} \mathrm{FeMo}_{1-x} \mathrm{~W}_{x} \mathrm{O}_{6}$ with $x=0.85$, whose magnetization data do not show appreciable pressure-induced effects, accordingly to the spin-glass insulating character of this system. ${ }^{9,10}$

\section{EXPERIMENTAL RESULTS}

The investigated samples, i.e., $\mathrm{Sr}_{2} \mathrm{FeMoO}_{6}$ (SFMO), $\mathrm{Ba}_{2} \mathrm{FeMoO}_{6}$ (BFMO), $\mathrm{Ca}_{2} \mathrm{FeMoO}_{6}$ (CFMO), $\mathrm{Sr}_{2} \mathrm{FeMo}_{0.3} \mathrm{~W}_{0.7} \mathrm{O}_{6} \quad$ (SW70), and $\mathrm{Sr}_{2} \mathrm{FeMo}_{0.15} \mathrm{~W}_{0.85} \mathrm{O}_{6}$ (SW85), were prepared by using the procedure reported in Ref. 24. The hydrostatic pressure was produced in a copperberyllium piston cylinder clamp, especially designed for magnetization measurements under pressure (see Ref. 25). The pressure cell was filled with the sample and Fluorinert FC77 as pressure transmitting medium. The pressure values were determined by measuring the shift of the superconducting transition temperature of a small piece of lead loaded together with the samples. The field cooling magnetization was measured with a commercial Superconducting Quantum Interference Device. The zero-pressure measurement could not be performed in the pressure cell since a small pressure had to be applied in order to seal the cell.

The normalized magnetization as a function of temperature at different pressure is shown in Fig. 1 for BFMO, SFMO, and CFMO, and in Fig. 2 for SW70 and SW85. The insets in Figs. 1 and 2 show the magnetization curves close to $T_{C}$. From the magnetization curves measured at the lowest pressure, $T_{C}$, was estimated by a linear extrapolation to zero magnetization [see inset in Fig. 1(a)]. The highest $T_{C}$ was found in SFMO at around $370 \mathrm{~K}$, and BFMO and CFMO exhibit similar $T_{C}$, around 305 and $315 \mathrm{~K}$, respectively. These values of $T_{C}$ are slightly lower, except for BFMO, than those reported in Ref. 8 (308, 385, and $365 \mathrm{~K}$ for BFMO, SFMO, and CFMO, respectively) and Ref. 7 (318, 404 , and $320 \mathrm{~K}$ for BFMO, SFMO, and CFMO, respectively). These differences may be due to various effects, such as the exact stoichiometry, cation mis-site disorder, and oxygen vacancies, which can have a strong impact on the value of the Curie temperature, ${ }^{26}$ but not on its pressure dependence. The present work is focused on the relative change in the Curie temperature induced by the external pressure, and thus the absolute values of $T_{C}$ are not essential for the present discussion.

The SW70 magnetization data show a very broad magnetic transition [see Figs. 2(a) and 2(b)], as a result of the loss of ferromagnetism with $\mathrm{W}$ doping. From a linear extrapolation, $T_{C}$ was estimated to be about $275 \mathrm{~K}$, with a large uncertainty due to the broadness of the transition. A broad cusp in the magnetization curve is present at low temperature (around $40 \mathrm{~K}$ ). Measurements performed on the spin-glass

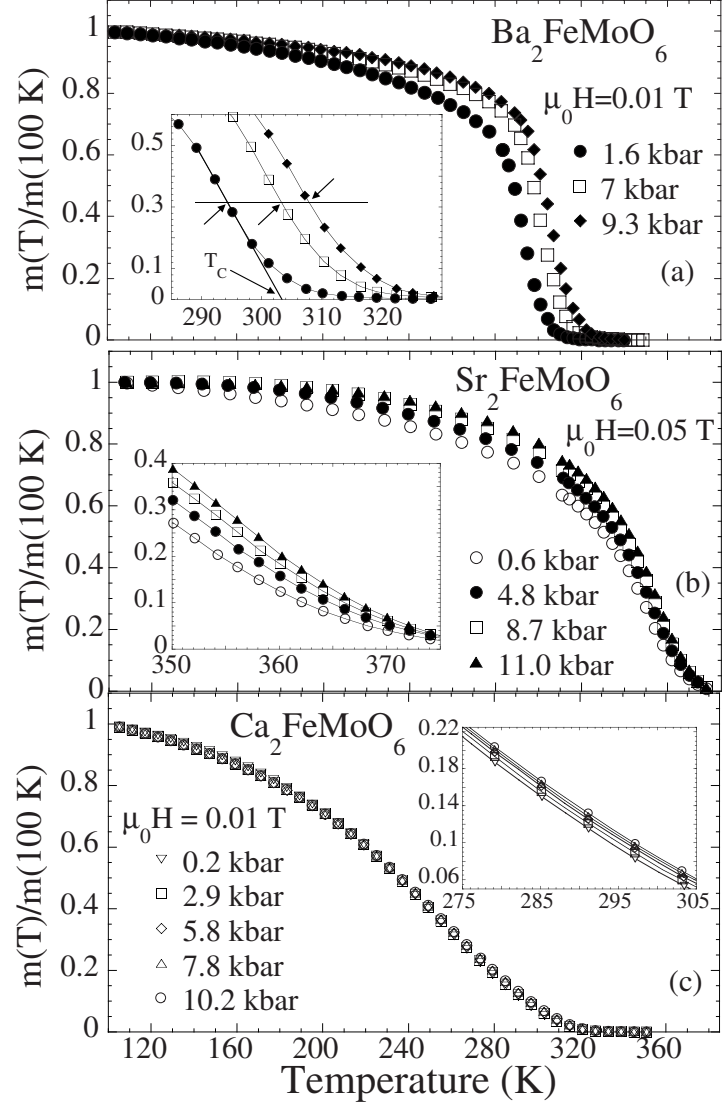

FIG. 1. Normalized magnetization as a function of temperature for $\mathrm{Ba}_{2} \mathrm{FeMoO}_{6}$ [panel (a)], $\mathrm{Sr}_{2} \mathrm{FeMoO}_{6}$ [panel (b)], and $\mathrm{Ca}_{2} \mathrm{FeMoO}_{6}$ [panel (c)] at different pressures. Insets: normalized magnetization for the three samples in the high-temperature region. The temperature range is slightly different for each sample in order to better show the pressure-induced curve shift. The lines are guides for the eye.

insulating system SW85 showed no shift of the magnetization curve with pressure, as expected, and clear evidence of a cusp at around $30 \mathrm{~K}$, much narrower than in the SW70 [see Fig. 2(c)]. We notice that Kobayashi et al. ${ }^{9}$ and Kawanaka et al. ${ }^{11}$ measured the temperature-dependent magnetization of the insulating antiferromagnet pristine $\mathrm{Sr}_{2} \mathrm{FeWO}_{6}$ (SFWO) sample, which shows a Curie-Weiss behavior with a peak at the antiferromagnetic ordering temperature $T_{N} \simeq 37 \mathrm{~K}$. The cusp in the low-temperature magnetization observed in SW85 [Fig. 2(c)], and-less markedly—in SW70 [Fig. 2(a)], can then be interpreted as a remanence of antiferromagnetism, as also proposed by Kobayashi and collaborators. ${ }^{9}$

The pressure has the largest effect on the BFMO sample, while the smallest effect is observed in CFMO, as it is shown in the insets of Fig. 1. Owing to the above mentioned possibility that the absolute values of the $T_{C}$ 's are affected by spurious effects and to the broadness of the transitions, we inferred the pressure dependence of $T_{C}$ from the temperature difference $\Delta T_{C}=T_{C}(\mathrm{p})-T_{C}\left(\mathrm{p}_{0}\right)\left(\mathrm{p}_{0}\right.$ indicates the lowest pressure for each sample) taken at a fixed magnetization value in the linear region close to the inflection point [see the arrows in the inset of Fig. 1(a)]. For the SFMO sample we measured $\Delta T_{C}$ for several values of the field $\left(\mu_{0} H=0.05,0.1\right.$, and $1 \mathrm{~T}$, 


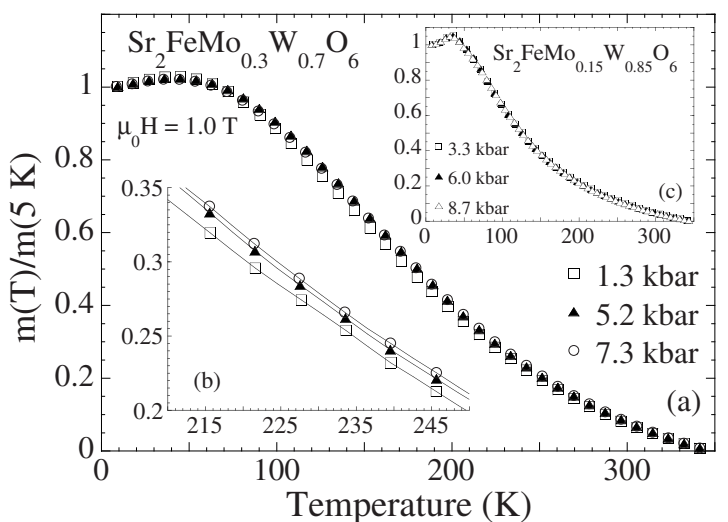

FIG. 2. (a) Normalized magnetization as a function of temperature for the $\mathrm{Sr}_{2} \mathrm{FeMo}_{0.3} \mathrm{~W}_{0.7} \mathrm{O}_{6}$ sample at different pressures. (b) Normalized magnetization for the same sample in the hightemperature region. (c) Normalized magnetization for the $\mathrm{Sr}_{2} \mathrm{FeMo}_{0.15} \mathrm{~W}_{0.85} \mathrm{O}_{6}$ sample at different pressures. The lines are guides for the eyes.

not shown). We found that, while $T_{C}$ changes with field, $\Delta T_{C}$ does not, at least within the experimental error. This finding allows us to compare $\Delta T_{C}$ obtained at different applied fields, namely, in BFMO and CFMO at $0.01 \mathrm{~T}$ and in SFMO at $0.05 \mathrm{~T}$ (see Fig. 1). In Fig. 3 we show $\Delta T_{C}$ as a function of pressure for the BFMO, SFMO, CFMO, and SW70 samples. The error bars on the temperature scale are given by the difference in temperature at constant magnetization obtained in repeated measurements on the same sample.

In all cases a linear fit well describes the observed pressure dependence of the Curie temperature (see Fig. 3). The rate $d T_{C} / d p$ depends on the system: CFMO shows the smallest rate $\left[d T_{C} / d p=0.25(8) \mathrm{K} / \mathrm{kbar}\right]$, being $d T_{C} / d p$ in SFMO $[0.60(8) \mathrm{K} / \mathrm{kbar}]$ and in BFMO [1.7(2) K/kbar] about two and seven times larger, respectively. The results for SFMO and BFMO are in very good agreement with the reported values in Refs. 18 and 19. In the SW70 system $T_{C}$ increases at a rate $\left[d T_{C} / d p=0.7(1) \mathrm{K} / \mathrm{kbar}\right]$ similar to that observed in SFMO.

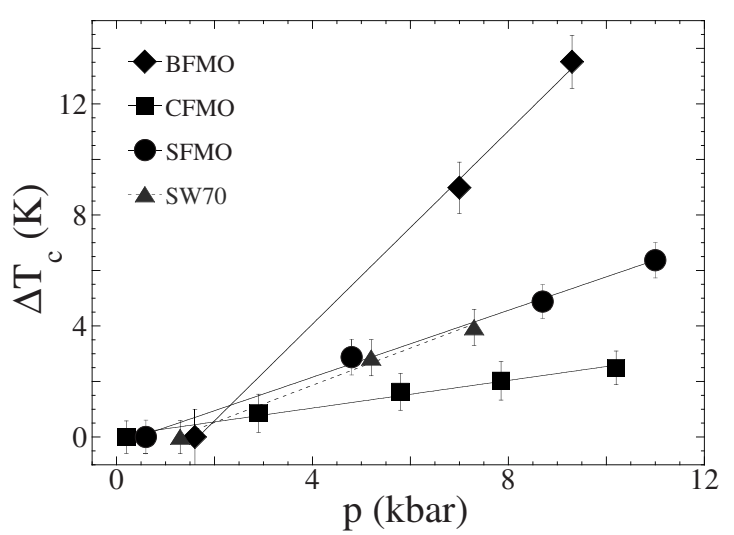

FIG. 3. $\Delta T_{C}$ (see text for definition) as a function of pressure for the three isoelectronic samples $\mathrm{Ba}_{2} \mathrm{FeMoO}_{6}, \mathrm{Sr}_{2} \mathrm{FeMoO}_{6}$, and $\mathrm{Ca}_{2} \mathrm{FeMoO}_{6}$, and for $\mathrm{Sr}_{2} \mathrm{FeMo}_{0.3} \mathrm{~W}_{0.7} \mathrm{O}_{6}$. The lines are linear fits to the data.

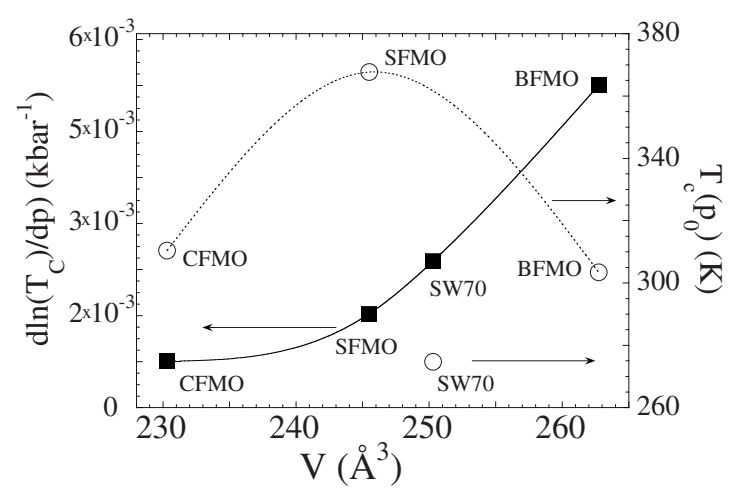

FIG. 4. $T_{C}$ at the lowest pressure (right axis, circles) and $d \ln \left(T_{C}\right) / d p$ (left axis, squares) for $\mathrm{Ba}_{2} \mathrm{FeMoO}_{6}, \mathrm{Sr}_{2} \mathrm{FeMoO}_{6}$, $\mathrm{Ca}_{2} \mathrm{FeMoO}_{6}$, and $\mathrm{Sr}_{2} \mathrm{FeMo}_{0.3} \mathrm{~W}_{0.7} \mathrm{O}_{6}$. The lines are guides for the eyes. The dotted line links the $T_{C}$ values of the isoelectronic samples (BFMO, SFMO, and CFMO).

\section{DISCUSSION AND CONCLUSIONS}

In order to discuss the presented results, we recall that BFMO, SFMO, and CFMO are formally isoelectronic, with a single electron occupying the spin-down subband formed by orbitals of $3 \mathrm{t}_{2 g}(\mathrm{Fe})+4 \mathrm{t}_{2 g}(\mathrm{Mo})+2 \mathrm{p}(\mathrm{O}){ }^{3}{ }^{3}$ Nevertheless, they show different Curie temperatures with a nonmonotonic behavior as a function of chemical pressure: $T_{C}(\mathrm{Sr})>T_{C}(\mathrm{Ba})$ and $T_{C}(\mathrm{Ca})$ (present work in Fig. 4 and Refs. 7, 8, and 20). In particular, it was shown ${ }^{7,22}$ that $T_{C}$ increases with increasing the chemical pressure from BFMO to SFMO, reaches a maximum for a slightly $\mathrm{Ca}$ substituted $\mathrm{Sr}_{2-x} \mathrm{Ca}_{x} \mathrm{FeMoO}_{6}$, and then decreases with further increasing the chemical pressure toward the pristine CFMO. Under the hypothesis of an equivalence between chemical and external pressure, we expect a high and positive $d T_{C} / d p$ in BFMO since by replacing $\mathrm{Ba}$ with $\mathrm{Sr}$ in BFMO, a strong increase in $T_{C}$ occurs. On the contrary, we expect a small and positive $d T_{C} / d p$ in SFMO since by replacing a small amount of $\mathrm{Sr}$ with $\mathrm{Ca}$ in SFMO, the $T_{C}$ slightly increases. ${ }^{7}$ As for CFMO, a small negative or, at least, a vanishingly small $d T_{C} / d p$ can be conjectured since in $\mathrm{Sr}_{2-x} \mathrm{Ca}_{x} \mathrm{FeMoO}_{6}, T_{C}$ slightly decreases on increasing the Ca content from $x \simeq 1$ to $x=2$, $^{7,22}$ Present results (see Fig. 3) do confirm the predictions for SFMO and BFMO contrary to the case of CFMO, for which a pressure-induced increase in $T_{C}\left(d T_{C} / d p>0\right)$, although small, has been observed.

We notice that, assuming the proportionality between $T_{C}$ and $\mathrm{BW}$, some authors ${ }^{7,8}$ tried to account for the chemical pressure dependence of $T_{C}$ in double perovskites using an approach which was successfully applied to describe perovskite $A B X_{3}$ compounds (particularly $\mathrm{AMnO}_{3}$ manganites) in a tight-binding approximation. ${ }^{27-29}$ Under this approximation the BW depends on both the $B-\mathrm{O}-B$ bond angles and $B-\mathrm{O}$ bond lengths, through the overlap integrals between the $3 d$ orbitals of the metal ion $B$ and the $2 p$ orbitals of the $\mathrm{O}$ anion. ${ }^{29}$ On these basis the dependence of $\mathrm{BW}$ on the bond angle $\theta$ and on the bond distance $d$ is provided by the empirical formula,

$$
\mathrm{BW} \propto \cos [(\pi-\theta) / 2] / d^{7 / 2} .
$$

On applying this model to the double-perovskite case, the differences in $T_{C}$ shown by the different SFMO, BFMO, and 
CFMO compounds might be ascribed to chemical-pressureinduced variations in the conduction BW. The bandwidth variation in the three samples was then estimated from the variation in the bond distance Fe-O-Mo $(d)$ and bond angle $(\theta)$. A nonmonotonic behavior of $\mathrm{BW}$ as a function of the $\mathrm{A}$ cation ionic size (chemical pressure) was found, quite similar to that of $T_{C}{ }^{8}$ Indeed, by substituting $\mathrm{Sr}$ with $\mathrm{Ca}$ the volume reduces, mainly due to a decrease in the Fe-O-Mo angle, rather than to a reduction in the bond lengths $(\mathrm{Fe}, \mathrm{Mo})-\mathrm{O}$, which indeed keeps almost constant. ${ }^{17,22}$ Thus, according to Eq. (1), BW decreases. On the other hand, for A cations larger than $\mathrm{Sr}$, i.e., the $\mathrm{Ba}$ ion, the structure is cubic; the Mo-O-Fe bond has an optimal overlapping $\left(180^{\circ}\right)$, but the unit cell expands due to the large size of the $\mathrm{Ba}$ ion, thus, reducing $\mathrm{BW}$. For the intermediate ion size $\mathrm{Sr}$, and a bond angle close to $180^{\circ}\left[174^{\circ}\right.$ (Ref. 8)], BW would reach the highest value. Thus, on a pure qualitative ground, the above manganitelike model appears to be successful in the present case. Since a number of criticisms have been raised in recent papers on the transferability of the model to doubleperovskite systems, ${ }^{21,30}$ we tried to check it on a more quantitative basis.

In order to verify quantitatively the validity of the formula in Eq. (1) we calculate $d \ln \left(T_{C}\right) / d p$ in the case of BFMO. ${ }^{31}$ We choose this system for calculations since it is cubic and retains the cubic structure even if half of the $\mathrm{Ba}$ ions is substituted with $\mathrm{Sr}$ ions. ${ }^{7,8}$ Therefore, we can expect that the application of an external pressure affects only the bond length $d$, which in a cubic structure is constrained to be $1 / 4$ of the lattice parameter $a{ }^{21}$ From Eq. (1) we thus can write (see also Ref. 31)

$$
\frac{d \ln \left(T_{C}\right)}{d p}=\frac{1}{T_{C}} \frac{d T_{C}}{d p}=3.5 k(d)=3.5 \frac{1}{a} \frac{d a}{d p},
$$

where $k(d)$ is the bond length compressibility. Given the bulk modulus $\mathrm{B}=2340 \mathrm{kbar}$ (see Ref. 15) and considering that $V=a^{3}$, we thus estimate $d \ln \left(T_{C}\right) / d p=4.97 \times 10^{-4} \mathrm{kbar}^{-1}$. From our experimental data we found $d \ln \left(T_{C}\right) / d p \simeq 5.7$ $\times 10^{-3} \mathrm{kbar}^{-1}$, about one order of magnitude higher than the above value, estimated on the basis of simple steric modifications of the bandwidth. A similar discrepancy was found by Laukhin and collaborators in manganites ${ }^{31}$ and ascribed to a remarkable pressure dependence of the electron-phonon coupling, ${ }^{32}$ not accounted for in Eq. (2). This argument does not apply to ordered double perovskites. Indeed, in these systems the electron-phonon coupling seems not to play a significant role in determining the Curie temperature. ${ }^{5}$ This statement is further supported by recent high-pressure Raman measurements, ${ }^{33}$ where the electron-phonon coupling is shown to be pressure independent up to $150 \mathrm{kbar}$, whereas the present results show a pressure dependence of $T_{C}$. On the other hand, Wojcik et al. ${ }^{21}$ in a recent NMR work claims that the formula in Eq. (1), based on a model of double-exchange manganites, is properly suited for bonds of $e_{g}$ symmetry and a unique metal-oxygen bond length, but it is not adequate to describe double-perovskites properties. Indeed, in double perovskites the relevant orbitals have a $t_{2 g}$ symmetry and two different bond lengths (Fe-O, Mo-O) are involved. Moreover, possible charge redistribution between $\mathrm{Mo}-4 d$ and Fe-3d, oc- curring when chemical pressure is applied by ion substitution, ${ }^{21}$ could also be responsible for additional effects when applying external pressure. The discrepancy between the present experimental value of $d \ln \left(T_{C}\right) / d p$ in BFMO, and the one estimated through Eq. (2) can be explained on the basis of all the above arguments. This also suggests a different nature of the magnetic interaction in doped manganites and in ordered double perovskites, as previously proposed. 5,21

To further discuss the whole of the present data and the unexpected pressure response of CFMO, we report in Fig. 4 the experimental $d \ln \left(T_{C}\right) / d p$ for BFMO, SFMO, CFMO, and SW70 as a function of the cell volume. $d \ln \left(T_{C}\right) / d p$ monotonically increases with increasing volume, regardless the lattice and/or the magnetic structures. On the contrary, the nonmonotonic behavior of the $T_{C}$ values as a function of the volume is quite clear in Fig. 4 . We notice that the $T_{C}$ value of the charge-doped SW70 significantly departs from the $T_{C}$ volume dependence of the isoelectronic compounds (see the dotted line in Fig. 4).

The behavior of $d \ln \left(T_{C}\right) / d p$ suggests that the sensitivity of $T_{C}$ to the external pressure depends on the possibility of the bond length to contract. Indeed, the BFMO sample has both a cubic structure, retained even if a large amount of $\mathrm{Sr}$ is substituted in the system, and a large volume, due to the large $\mathrm{Ba}$ ionic size, leading to a large space available for Mo and $\mathrm{Fe}$ ions. This means that the structure has large possibility to contract just by decreasing the bond length, thus, increasing the bandwidth. Therefore, a large variation in $T_{C}$ with pressure is expected. To allocate the smaller $\mathrm{Sr}$ ion, a contraction of the bond length is not enough, and the structure get slightly distorted with a decrease in the bond angle, with respect to BFMO, to reduce the volume. This suggests that a further contraction of the bond length by applying an external pressure is more difficult to achieve, and $d \ln \left(T_{C}\right) / d p$ is remarkably smaller. The $\mathrm{W}$-substituted SW70 sample has a bit larger volume than the pristine SFMO sample, giving a slightly larger $d \ln \left(T_{C}\right) / d p$. By substituting $\mathrm{Sr}$ with $\mathrm{Ca}$ no substantial change in the bond length was observed, whereas the bond angle noticeably changes, leading to a dramatic change in lattice structure. ${ }^{17,22}$ An additional external pressure, therefore, can hardly modify the bond length in the contracted and distorted CFMO system, thus, inducing a very small, but nonzero, positive change in $T_{C}$.

We notice that a departure from the hydrostatic-chemical pressure equivalence can be inferred also from a critical analysis of the data previously reported in the literature. Indeed, the stability under external pressure of both the BFMO and the SFMO structures has been proved up to a volume compression of about $10 \%,{ }^{15,16}$ whereas a volume compression of about $6 \%$ induced by chemical pressure on going from SFMO to CFMO (see Fig. 4) causes a change in the lattice symmetry. The cage compression for BFMO and SFMO (cubic and pseudocubic structures, regular and slightly in plane distorted octahedra, respectively) indeed can be obtained through an isotropic volume reduction in the octahedral units, which weakly affects the structural symmetry. This kind of compression is not possible for CFMO which has the lowest symmetry and fully distorted octahedra (three different bond lengths). 
From the all the above considerations we can schematically conclude that: (i) external pressure always induces an increase in $T_{C}$; (ii) in BFMO and SFMO the increase in $T_{C}$ with chemical pressure is qualitatively similar to that one induced by the external pressure (large in BFMO and small in SFMO); (iii) the equivalence between chemical and external pressure does not hold when a chemical-pressure-induced structural transition does occur, as what happens in the present case when $\mathrm{Sr}$ is replaced by $\mathrm{Ca}$.

In summary, we performed a systematic study of the pressure dependence of the Curie temperature $T_{C}$ in a group of double perovskites: the isoelectronic compounds $\mathrm{Sr}_{2} \mathrm{FeMoO}_{6}, \mathrm{Ba}_{2} \mathrm{FeMoO}_{6}$, and $\mathrm{Ca}_{2} \mathrm{FeMoO}_{6}$, in which different chemical pressures are present, and $\mathrm{W}$-substituted $\mathrm{Sr}_{2} \mathrm{FeMo}_{1-x} \mathrm{~W}_{x} \mathrm{O}_{6}$ with $x=0.70$. We notice that the pressure effects observed in the CFMO and in the W70 compounds, not previously reported, are crucial in the present paper. The CFMO compound largely extends the range of chemical pressure exploited by chemical substitution and exhibits most of the unpredicted pressure effects presently observed.
The W70 compound, which enables charge doping, allows studying combined modifications of lattice and electronic degree of freedom. Magnetization measurements under pressure revealed an increase in $T_{C}$ with pressure in all the investigated samples. The different rates of increase so obtained can be ascribed to the different lattice symmetry and volume cell, which influence the possibility of the bond length to contract. The analysis of the present data, combined with the findings of a pressure-independent electron-phonon coupling, ${ }^{33}$ also suggests a different nature of the magnetic interaction in ordered double perovskites with respect to the doped manganites, in agreement with previous results ${ }^{5,21}$

\section{ACKNOWLEDGMENTS}

We are grateful to N. L. Saini for stimulating discussions on the subject. This work was partly supported by the EU Project CoMePhS, by the K. Alex Müller Foundation, and by the Department of Science and Technology, Government of India and DAE-BRNS.
${ }^{1}$ F. S. Galasso, F. C. Douglas, and R. J. Kasper, J. Chem. Phys. 44, 1672 (1966).

${ }^{2}$ R. von Helmolt, J. Wecker, B. Holzapfel, L. Schultz, and K. Samwer, Phys. Rev. Lett. 71, 2331 (1993); For a review: Phys. Today 48 (4) (1995), Special issue: Magnetoelectronics.

${ }^{3}$ K. I. Kobayashi, T. Kimura, H. Sawada, K. Terakura, and Y. Tokura, Nature (London) 395, 677 (1998).

${ }^{4}$ S. A. Wolf, D. D. Awschalom, R. A. Buhrman, J. M. Daughton, S. von Molnar, M. L. Roukes, A. Y. Chtchelkanova, and D. M. Treger, Science 294, 1488 (2001).

${ }^{5}$ D. D. Sarma, P. Mahadevan, T. Saha-Dasgupta, S. Ray, and A. Kumar, Phys. Rev. Lett. 85, 2549 (2000).

${ }^{6}$ J. B. Philipp, P. Majewski, L. Alff, A. Erb, R. Gross, T. Graf, M. S. Brandt, J. Simon, T. Walther, W. Mader, D. Topwal, and D. D. Sarma, Phys. Rev. B 68, 144431 (2003).

${ }^{7}$ Bog-Gi Kim, Yew-San Hor, and S.-W. Cheong, Appl. Phys. Lett. 79, 388 (2001).

${ }^{8}$ C. Ritter, M. R. Ibarra, L. Morellon, J. Blasco, J. García, and J. M. De Teresa, J. Phys.: Condens. Matter 12, 8295 (2000).

${ }^{9}$ K. I. Kobayashi, T. Okuda, Y. Tomioka, T. Kimura, and Y. Tokura, J. Magn. Magn. Mater. 218, 17 (2000).

${ }^{10}$ R. I. Dass and J. B. Goodenough, Phys. Rev. B 63, 064417 (2001).

${ }^{11}$ H. Kawanaka, I. Hase, S. Toyama, and Y. Nishihara, J. Phys. Soc. Jpn. 68, 2890 (1999)

${ }^{12}$ T. Okuda, K.-I. Kobayashi, Y. Tomioka, and Y. Tokura, Phys. Rev. B 68, 144407 (2003).

${ }^{13}$ J.-S. Kang, J. H. Kim, A. Sekiyama, S. Kasai, S. Suga, S. W. Han, K. H. Kim, T. Muro, Y. Saitoh, C. Hwang, C. G. Olson, B. J. Park, B. W. Lee, J. H. Shim, J. H. Park, and B. I. Min, Phys. Rev. B 66, 113105 (2002).

${ }^{14}$ A. Poddar, S. Das, and B. Chattopadhyay, J. Appl. Phys. 95, 6261 (2004).

${ }^{15}$ R. C. Yu, P. Zhao, F. Y. Li, Z. X. Liu, Z. Zhang, J. Liu, and C. Q. Jin, Phys. Rev. B 69, 214405 (2004).

${ }^{16}$ P. Zhao, R. C. Yu, F. Y. Li, and Z. X. Liu, J. Appl. Phys. 92, 1942 (2002).

${ }^{17}$ T. S. Chan, R. S. Liu, G. Y. Guo, S. F. Hu, J. G. Lin, J. M. Chen, and J. P. Attfield, Chem. Mater. 15, 425 (2003).

${ }^{18}$ T. Goko, Y. Endo, E. Morimoto, J. Arai, and T. Matsumoto, Physica B (Amsterdam) 329-333, 837 (2003).

${ }^{19}$ R. Wang and M. Itoh, Solid State Ionics 108, 269 (1998).

${ }^{20}$ R. P. Borges, R. M. Thomas, C. Cullinan, J. M. D. Coey, R. Suryanarayanan, L. Ben-Dor, L. Pinsard-Gaudart, and A. Revcolevschi, J. Phys.: Condens. Matter 11, L445 (1999).

${ }^{21}$ M. Wojcik, E. Jedryka, S. Nadolski, D. Rubi, C. Frontera, J. Fontcuberta, B. Jurca, N. Dragoe, and P. Berthet, Phys. Rev. B 71, 104410 (2005).

${ }^{22}$ C. Ritter, D. Rub, J. Navarro, C. Frontera, J.-L. Garcia-Munoz, and J. Fontcuberta, J. Magn. Magn. Mater. 272-276, 852 (2004).

${ }^{23}$ W. Zhong, W. Liu, X. L. Wu, N. J. Tang, W. Chen, C. T. Au, and Y. W. Du, Solid State Commun. 132, 157 (2004).

${ }^{24}$ Sugata Ray, Ashwani Kumar, Subham Majumdar, E. V. Sampathkumaran, and D. D. Sarma, J. Phys.: Condens. Matter 13, 607 (2001).

${ }^{25}$ T. Strässle, Ph.D. thesis, ETH Zürich, 2001.

${ }^{26}$ A. S. Ogale, S. B. Ogale, R. Ramesh, and T. Venkatesan, Appl. Phys. Lett. 75, 537 (1999).

${ }^{27}$ P. G. Radaelli, G. Iannone, M. Marezio, H. Y. Hwang, S.-W. Cheong, J. D. Jorgensen, and D. N. Argyriou, Phys. Rev. B 56, 8265 (1997).

${ }^{28}$ M. Medarde, J. Mesot, P. Lacorre, S. Rosenkranz, P. Fischer, and K. Gobrecht, Phys. Rev. B 52, 9248 (1995).

${ }^{29}$ W. A. Harrison, The Electronic Structure and Properties of Solids (Freeman, San Francisco, 1980).

${ }^{30}$ J. M. De Teresa, D. Serrate, J. Blasco, M. R. Ibarra, and L. Morellon, Phys. Rev. B 69, 144401 (2004).

${ }^{31}$ V. Laukhin, J. Fontcuberta, J. L. García-Munõz, and X. Obradors, Phys. Rev. B 56, R10009 (1997).

${ }^{32}$ P. Postorino, A. Congeduti, P. Dore, A. Sacchetti, F. A. Gorelli, L. Ulivi, A. Kumar, and D. D. Sarma, Phys. Rev. Lett. 91, 175501 (2003)

${ }^{33}$ D. Marrocchelli, P. Postorino, D. Di Castro, E. Arcangeletti, P. Dore, M. C. Guidi, S. Ray, and D. D. Sarma, Phys. Rev. B 76, 172405 (2007). 